\title{
Diversidad cultural: análisis de su atención pedagógica en la práctica educativa en la primera infancia
}

\author{
Cultural diversity: an analysis \\ of pedagogical attention in early \\ childhood educational practice
}

\section{Carmen Andrea Aracena Lobos}

Universidad Finis Terrae

\section{RESUMEN}

En la actualidad se busca incorporar estrategias que atiendan a la diversidad cultural en la educación inicial. El objetivo principal de esta investigación fue comprender la atención pedagógica a la diversidad cultural en un nivel de sala cuna. La metodología utilizada fue la investigación-acción, con recolección de datos a partir de las técnicas de observación participante y entrevista semiestructurada. Los resultados sobre las acciones implementadas dieron cuenta de un reconocimiento y valoración de la diversidad cultural, así como también de la necesidad de dar continuidad a las acciones realizadas. La investigación permitió concluir que las acciones implementadas lograron atender pedagógicamente a la diversidad cultural del aula y la evaluación realizada sirvió para iniciar un nuevo proceso de investigación-acción.

Palabras clave: cultura, educación intercultural, escuela de párvulos, educación de la primera infancia 


\begin{abstract}
Nowadays there is a search to incorporate strategies that address cultural diversity in Early Childhood Education. The main objective of this research was to understand the pedagogical attention to cultural diversity at a nursery level. The methodology used was of research-action, with data collection based on participant observation techniques and semistructured interviews. The results on the actions implemented showed recognition and appreciation of the cultural diversity, as well as the need to give continuity to the actions carried out. The research led to conclude that the implemented actions achieved to pedagogically address the cultural diversity of the classroom and the evaluation conducted served to start a new process of research-action.
\end{abstract}

Keywords: culture, intercultural education, nursery school, early childhood education

\title{
INTRODUCCIÓN
}

La presente investigación se planteó con el propósito de comprender la atención pedagógica ofrecida a la diversidad cultural, en función de la realidad observada en el contexto de la práctica profesional. Se desarrolla en el período marzo-noviembre de 2019, en el aula de una sala cuna menor, compuesta por 17 niños y niñas (de entre 7 meses y 1 año 6 meses de edad) a cargo de un equipo pedagógico formado por una educadora de párvulos y tres asistentes técnicos.

En el aula se evidenció que prevalece la ausencia de experiencias pedagógicas que reconozcan y/o atiendan la diversidad cultural, de lo cual podría inferirse que hay factores actitudinales, procedimentales y conceptuales que demuestran la ausencia de herramientas para abordar pedagógicamente la diversidad cultural presente en la sala.

\section{Diversidad}

Educar para la diversidad supone tener la capacidad de reconocer que los niños y niñas tienen distintas necesidades y características 
propias que se han generado a través de distintos factores (biológicos y ambientales) que han influido en su desarrollo y aprendizaje (Fermín, 2007). Esta diversidad se entiende hoy desde una mirada compleja y amplia, en la cual se considera a personas en situación de discapacidad, de diferentes razas y etnias, seguidores de diversas religiones, a quienes eligen su sexualidad, los que tienen diversas posturas ideológicas en momentos históricos, es decir, todas aquellas que se pueden visualizar como disminuidas en determinadas situaciones. Es por ello que, para dar respuesta a esta diversidad, se requiere tener flexibilidad en el diseño y aplicación de experiencias en los centros educativos (Fermín, 2010).

La equidad se enfoca en el proceso de garantizar una distribución justa de bienes y servicios (Grupo Consultivo de Atención y Desarrollo de la Primera Infancia, 2016). A su vez, la diversidad se entiende como la pluralidad de significados que generamos y que compartimos en un determinado grupo, es decir, la variedad de formas de estar, ver y construir el mundo, la realidad y las relaciones con los demás (Márquez et al., 2017). En este sentido, una distribución justa de los bienes educativos implica tener en cuenta la diversidad de necesidades de las personas, de los grupos y de los ambientes. Una de las tantas manifestaciones de la diversidad en educación tiene que ver con la diversidad cultural.

\section{Diversidad cultural}

La cultura va tomando diversas formas a lo largo del tiempo y de los espacios. Esta diversidad se manifiesta en la originalidad y la pluralidad, y caracteriza a los grupos y las sociedades que componen la humanidad. Se toma como una fuente de intercambios, de innovación y de creatividad, forma el patrimonio común de la humanidad y se debe reconocer y ser consolidada (UNESCO, 2001).

En Chile, el año 2007 se promulga el acuerdo de la Convención sobre la Protección y Promoción de la Diversidad de las Expresiones Culturales, mediante el decreto n. ${ }^{\circ} 82$ del Ministerio de Relaciones 
Exteriores. La Convención reconoce la diversidad cultural como las formas de expresión de distintos grupos y sociedades, que exhiben la diversidad a través de diversos modos de creaciones artísticas, producciones, distribuciones, difusiones y disfrute de las expresiones culturales, y no solo en las formas en que se expresa, enriquece y transmite el patrimonio cultural (Consejo Nacional de la Cultura y las Artes, 2014).

A lo largo de los años se ha redefinido el concepto de diversidad cultural. Ibáńez-Salgado et al. (2012) demuestran que este concepto es parte de un marco social democrático y de relaciones entre distintas culturas, por lo que su reconocimiento se configura desde las construcciones de sentido que se generan en los procesos de socialización primaria, en los cuales los niños/as van formando un sistema de conocimientos enmarcado en sentidos propios y característicos de la cultura a la cual pertenecen, con significados que pueden ampliarse y modificarse.

La cultura se ve reflejada en lo que realizamos diariamente, ya sea desde el lenguaje que usamos, lo que vestimos, nuestra alimentación, hasta la actitud con la que nos enfrentamos a diversas situaciones y a las relaciones interpersonales. En el pasado no había parecido tan importante reafirmar y promover la propia identidad cultural y el entendimiento intercultural a través de la educación, como se hace actualmente. Con los avances de la globalización se ha producido una reunión de diversos estilos de vida, pero no ha significado una verdadera alianza entre ellos (Cuello, 2016), lo cual ha representado nuevos desafíos pedagógicos que exigen abordarla.

La cultura es el conjunto de elementos simbólicos, económicos, materiales, que marcan las actuaciones sociales y familiares de un individuo; además, se ve influida por el desarrollo histórico y por la educación recibida a lo largo de la vida. Desde esta comprensión surge el término de pluriculturalidad, entendido como la presencia simultánea de dos o más culturas en un territorio y su interrelación (Bernabé, 2012). 
En la actualidad se reconoce la diversidad como fenómeno cultural, lo que permite considerar la sociedad de este siglo desde la complejidad. Esto ha llevado a exigir respeto por la diversidad cultural más allá de los pueblos originarios o inmigrantes, considerando también las condiciones socioeconómicas, la orientación sexual, el género, las nuevas formas de familia, entre otros factores culturales (Fermín-Gonzáles, 2021). La cultura se refiere al conjunto de prácticas, conductas, representaciones y estilos de relación en un grupo humano, cuyo conocimiento, creencias y valores se trasmiten socialmente de generación en generación; y la diversidad es el resultado de modos distintos de construir significados, que dan lugar a una visión de mundo diversa que se forma según el modo de convivencia propio de cada cultura (Ibáńez-Salgado, 2015).

\section{Convivencia entre culturas}

Chile es un país pluricultural en el que conviven pueblos originarios, la comunidad afrodescendiente de Arica y Parinacota y una gran diversidad de otras culturas, como resultado de múltiples flujos migratorios (Consejo Nacional de la Cultura y las Artes, 2016).

Los pueblos indígenas u originarios son los pueblos descendientes de las agrupaciones humanas que existen en el territorio nacional, que conservan manifestaciones étnicas y culturales propias (Instituto Nacional de Estadísticas, 2018). De acuerdo con el Censo 2017, un $12,8 \%$ de la población encuestada se considera perteneciente a algún pueblo indígena u originario. Las personas de pueblos originarios se distribuyen a lo largo del país, concentrándose en la Región Metropolitana (Instituto Nacional de Estadísticas, 2017). En el año 2020, un 43,5\% de los hogares del país tienen niños, niñas y adolescentes, de los cuales un $12,9 \%$ pertenecen a un pueblo indígena y un $2,8 \%$ son inmigrantes (UNICEF, 2020).

La migración es una de las manifestaciones de la diversidad cultural, por lo que esta no niega que exista una diversidad propia dentro del país, expresada en las distintas familias y comunidades chilenas en 
las cuales crecen los niños y niñas. La migración es entendida como un "proceso, en la medida que el traslado de las personas desde un país a otro implica un conjunto de factores causales, de desarrollo y consecuencias, tanto para la sociedad de origen como la de destino" (Cano y Soffia, 2009, p. 2).

Se ha estudiado la migración interna, que se refiere al desplazamiento migratorio de personas dentro del mismo país. Además, "se considera la movilidad según siete tipos de flujo: internacional, interna, rural-urbana, urbana-urbana, circulación, o movilidad cotidiana, migración potencialmente suplantada por movilidad cotidiana y migración potencialmente sustituida por sistemas de circulación" (Chávez et al., 2016, p. 10). Escolano et al. (2018) exponen que las migraciones interurbanas no han sido objeto de reflexión teórica ni de análisis empíricos tan amplios; sin embargo, rescatan que en Chile existe predominio de los movimientos migratorios ciudad-ciudad e interurbanos.

En Chile, desde el año 1990 se intensifica la llegada de inmigrantes desde países de América Latina y el Caribe, atraídos por la estabilidad política y el crecimiento de la economía de nuestro país (Naudon, 2016). La llegada de extranjeros ha contemplado también niños, niñas y jóvenes en edad escolar, por lo que ha aumentado el número de estudiantes extranjeros en las aulas chilenas. Esto ha exigido que el sistema escolar sea abierto y flexible ante las necesidades sociales, lo que también requiere de políticas públicas pertinentes para contribuir de forma exitosa a la integración de estos estudiantes (Barrios y Palou, 2014).

Este cambio generado en la sociedad chilena ha producido quiebres en la relación de los equipos educativos y las familias, ya que algunos educadores han adoptado una posición pesimista frente al rol que desempeñan las familias tanto en la labor formativa como en la crianza de los niños y niñas, sin tener en cuenta las condiciones sociales y económicas que enfrentan estas y que han afectado el equilibrio del núcleo familiar (Moreno et al., 2016). 
La presencia y aumento de personas extranjeras en Chile ha tenido también un efecto sobre el sistema escolar nacional en todos los niveles y dependencias administrativas. En Educación Parvularia, en 2015, el nivel de escolaridad en curso o alcanzado según nacionalidad en el nivel sala cuna fue de un total de 77.966 niños y niñas chilenos y extranjeros asistentes a Jardín Infantil, de los cuales 603 niños y niñas eran extranjeros (Fernández, 2018).

En la Región Metropolitana se concentra la mayor cantidad de población extranjera a nivel nacional, junto con Arica y Parinacota, Tarapacá y Antofagasta. Estas cuatro regiones agrupan el 84\% de toda la población extranjera presente en Chile en el año 2015. Han aumentado su presencia en Chile migrantes de Perú, Bolivia, Venezuela, Haití y Ecuador. La situación actual de emigración de latinoamericanos y caribeños muestra que existen alrededor de unos 30 millones de latinoamericanos y caribeños residiendo en países distintos al de su nacimiento, lo que corresponde aproximadamente a un 4\% de la población total de América Latina y el Caribe (Martínez et al., 2014). Conocer estos datos permite inferir la repercusión que ello está teniendo en el ámbito educativo inicial, ya que la mayoría se moviliza junto a sus niños y niñas, generando así desafíos pedagógicos que ameritan ser atendidos.

\section{Diversidad cultural y educación inicial: desafío pedagógico en Chile}

La diversidad cultural está presente en todas las aulas, por lo que se hace necesario fortalecer los procesos de profesionalidad docente asumiendo el desafío de políticas educativas orientadas a la atención de la diversidad cultural desde una perspectiva de educación intercultural crítica (Mora, 2018). El estudio realizado por Barrero y Rosero (2018) da cuenta de las diversas concepciones de los educadores sobre qué y cómo se trabaja la diversidad en el campo de la educación inicial, dejando en claro cuatro atribuciones fundamentales, a través de sus estudios e investigaciones, las cuales reflejan que el $35 \%$ de los 
participantes atribuye la diversidad a la condición de estudiantes extranjeros e inmigrantes, el $25 \%$ a la condición de estudiantes en condición de discapacidad, el 15\% a la condición de estudiantes étnicos, específicamente población indígena, y, finalmente, un $25 \%$ a toda la población educativa sin ninguna condición específica.

En las Bases Curriculares de Educación Parvularia se establece que uno de sus fines es "propiciar aprendizajes de calidad en las niñas y los niños que sean pertinentes y consideren las necesidades educativas especiales, las diversidades culturales, lingüísticas, de género, religiosas y sociales, junto a otros aspectos culturales significativos de ellos..." (Ministerio de Educación, 2018).

La realidad educativa actual a nivel nacional e internacional evidencia dificultades para hacer efectiva la participación de las familias en los centros educativos. Existen dificultades para adaptarse a las nuevas necesidades de las familias, por lo que se requieren cambios para lograr este objetivo. Los canales de comunicación entre la familia y la escuela siguen reproduciendo esquemas tradicionales en los que la familia y la escuela mantienen una relación de desigualdad, lo que genera relaciones de competencia (Gomila y Pascual, 2015).

Es relevante conocer sobre las prácticas de crianza en cada contexto familiar; ello permitirá comprender el tipo de vinculación con la familia, acompañar las acciones de formación y educación, orientar y corregir en caso de ser necesario, valorando el papel que desempeña la crianza desde una concepción pedagógica. En el proceso de formación de los niños y niñas, el papel de los padres es fundamental en todas las culturas (Fermín, 2018).

\section{Educación intercultural}

La educación intercultural propone una práctica educativa que sitúe las diferencias culturales de individuos y grupos como foco de la reflexión y la indagación en los espacios educativos. Tiene como objetivo principal dar respuesta a la diversidad cultural propia de las sociedades democráticas desarrolladas, que respetan y valoran 
el pluralismo cultural como algo indispensable en las sociedades actuales y que se contempla como riqueza y recurso educativo (Arroyo, 2013). En este estudio, se buscó reflexionar e indagar en el espacio educativo para dar respuesta a la diversidad cultural propia del aula, generando acciones que permitieran la atención pedagógica de esta.

Al ingresar los nińos y nińas a los centros educativos se genera un conjunto de expectativas y concepciones por parte de las familias y de los educadores, lo que provoca diferentes formas de relación entre los padres y los docentes. Los establecimientos reciben en forma conjunta a los nińos/as y a sus familias, por lo que la construcción del vínculo entre la escuela y la familia se produce desde el ingreso. Las expectativas se definen como la anticipación de una relación en una situación futura (Llugain, 2015).

Según lo señalado, es crucial la percepción que tienen los docentes sobre temas referidos a la multiculturalidad e interculturalidad y globalización, ya que estos determinan el papel que desempeñan en el aula (Aranda, 2011). Por esto es que se buscó trabajar en conjunto con el equipo educativo para tomar decisiones pertinentes y mejorar la atención a las familias.

\section{Trabajo con la familia en educación parvularia}

Los referentes nacionales han hecho énfasis en el trabajo de la interculturalidad con los niños, niñas y la comunidad educativa. En las Bases Curriculares de Educación Parvularia se señala que para lograr que los ambientes de aprendizaje sean significativos, es importante que se relacionen con las características, necesidades e intereses de los niños y niñas, considerando las experiencias previas que poseen, las fortalezas que los potencian y el contexto sociocultural, intercultural y lingüístico al que pertenecen (Ministerio de Educación, 2018). Por su parte, Fundación Integra (2018) visualiza la interculturalidad desde un enfoque integral y de derechos humanos, refiriéndose a la construcción de relaciones equitativas entre personas, comunidades, países y culturas, lo que implica trabajar la interculturalidad desde 
una perspectiva que incluya elementos históricos, sociales, culturales, políticos, económicos, educativos, antropológicos, ambientales, entre otros. Finalmente, la Junta Nacional de Jardines Infantiles (2009) indica que, para favorecer una mejor adaptación de los niños y niñas en los primeros meses, se deben considerar algunos de los patrones de crianza de las familias y la historia de vida de cada uno, incorporando a las rutinas diarias elementos de su cultura particular que se vinculan con la etapa de vida en la que se encuentran: expresiones afectivas en su lengua materna, pautas de crianza familiares, alimentos, preparaciones y costumbres asociadas a la satisfacción de sus necesidades básicas.

En mayo de 2015 se promulga la Ley de Inclusión Escolar (n. 20.845), que involucra transformaciones al sistema de educación chileno. En ella se hace alusión a la diversidad y se establece que el sistema debe promover y respetar la diversidad cultural, religiosa y social de las familias que han elegido un proyecto diverso y determinado, y que son atendidas por él, en conformidad a la Constitución y las leyes. En los establecimientos educacionales de propiedad o administración del Estado se promoverá la formación laica, esto es, respetuosa de toda expresión religiosa, y la formación ciudadana de los estudiantes, a fin de fomentar su participación en la sociedad (Ministerio de Educación, 2017).

El Marco para la Buena Enseñanza de Educación Parvularia promueve interacciones pedagógicas que consideran y valoran la interculturalidad, la cual se entiende como un proceso dinámico que favorece la creación de nuevas formas de convivencia ciudadana entre todos, sin distinción de nacionalidad u origen. Se reconoce y valora la riqueza de la diversidad lingüístico-cultural, natural y espiritual (Ministerio de Educación, 2019).

En función de lo expuesto anteriormente, desde la contingencia respecto de esta temática, se planteó como objetivo general analizar el proceso de atención pedagógica ofrecida a la diversidad cultural en el aula, buscando específicamente identificar prácticas de atención a la diversidad cultural, para posteriormente diseñar acciones, implementarlas y luego evaluarlas. 


\section{METODOLOGÍA}

La investigación se abordó a través del paradigma sociocrítico, el cual se orienta a la práctica educativa en función de generar cambios como resultado de una toma de decisiones consciente. La práctica investigativa se caracteriza por una acción-reflexión-acción, e implica que el investigador se proponga generar un cambio en un cierto contexto social. Busca la transformación social, basándose en la participación, intervención y colaboración desde la reflexión personal crítica en la acción (Ramos, 2015).

La investigación-acción procura la consideración de todos los miembros de la comunidad como "socios" y los valora por sus perspectivas únicas y la información que proporcionan durante todo el proceso, por lo que el estudio es conducido con la comunidad más que en una comunidad. Se debe conocer a la comunidad en toda su complejidad, más que solamente identificar sus necesidades (Hernández et al., 2014).

Para esta investigación se consideró al equipo educativo de la sala cuna menor (1 educadora y 3 asistentes técnicos) y apoderados de los niños y niñas (17 niños y niñas entre los 7 meses de edad y 1 año 6 meses), pertenecientes a un centro educativo que atiende a nińos y niñas entre 0 a 4 años con funcionamiento por Vía Transferencia de Fondos, es decir, administrado por una organización sin fines de lucro, perteneciente a una corporación educacional.

El diseńo del trabajo responde a una investigación cualitativa. Este modelo es flexible y emergente, "implica volver de adelante a atrás, de lo más concreto a lo más abstracto, de citas a macrointerpretaciones, en un ir y venir que tiene que realizarse en forma programada y también emergente durante todo el proceso" (Echeverría, 2005, p. 9). Para ello, se utilizó la investigación-acción, la cual busca aportar información que guíe la toma de decisiones y los procesos de cambio para la mejora de la misma (Sandin, 2003). Se seleccionó este tipo de investigación debido a que tomó parte en ella una educadora en formación activa en el contexto del aula, lo que permitió reflexionar 
sobre la propia praxis y actuar en consecuencia para conseguir las transformaciones, buscando tomar decisiones en conjunto con la comunidad educativa para mejorar las prácticas que se pudieron evidenciar en ella. En la figura 1 se explicita el proceso seguido.

1. Fase diagnóstica del problema de investigación
2. Fase diseño del plan de intervención
3. Fase implementación del plan de acción

Fuente: Adaptado de Sandin (2003)

Fases de la investigación-acción y actividades en cada una de ellas

FASE I. Diagnóstico del problema de investigación. Para llevar a cabo el diagnóstico de la problemática de la sala cuna menor, se realizó una observación sistemática. Como resultado de este trabajo se pudo observar la presencia de familias de diversas nacionalidades: chilenas provenientes de Santiago (47\%), de las cuales ninguna se declara perteneciente a pueblos indígenas u originarios; haitianas $(35,2 \%)$; peruanas $(11 \%)$; y colombianas $(5,8 \%)$. Debido a esto, se decidió enfocar la mirada en el abordaje pedagógico de las diferencias culturales presentes en la sala; sobre este particular se observaron algunas iniciativas muy incipientes para atender la diversidad cultural por parte del equipo educativo, lo que permitió plantear el problema de investigación.

FaSE II. Diseño del plan de intervención. Se diseñó un plan de intervención para la mejora correspondiente a diversos ámbitos: comunitario, de desarrollo curricular y metodológico, y de desarrollo profesional/formación. Además, de cada acción derivaron numerosas tareas y, en conjunto con el equipo educativo, se les dio un orden de prioridad. 


\section{FASE III. Implementación del plan de acción diseñado. A} continuación, se presenta la implementación del plan de acción diseñado con 8 tareas ${ }^{1}$.

- TAREA 1. Diccionario creol-español. Se realizó la adaptación del cuadernillo "Hablemos espańol, cuadernillo creol haitianoespañol con información práctica sobre Chile", de Fuenzalida et al., (2017). Este sirvió para poder conocer léxico y facilitar la comunicación con los ciudadanos haitianos.

- TAREA 2. Caja de avisos de orientación y atención pedagógica a comunidad migrante. Se elaboró una caja con hojas en formato flyer, que contenían diversos tipos de avisos dirigidos a la comunidad.

- TAREA 3. Mapa con los países de origen de las familias del aula. Se imprimió un mapa de América y en él se destacaron los países de origen de las familias del aula; en este caso, Perú, Colombia, Haití y Chile.

- TAREA 4. Cuaderno viajero. Se elaboró un cuaderno con el título "Nuestra familia/fanminou", para que las familias plasmaran parte de su historia familiar, sus gustos en común, actividades preferidas, la música que escuchan, entre otros.

- TAREA 5. El jardín que soñamos. Se pusieron hojas en blanco en un atril con un lápiz a libre disposición, y se invitó a las familias, a través del título "El jardín que soñamos", a que plasmaran sus deseos como comunidad.

- TAREA 6. Taller de crianza. Se realizó un taller de crianza solo con una madre del aula, debido a la contingencia nacional. En este espacio pudo exponer sus desafíos, inquietudes y contar

1 Algunas de las tareas debieron ser reorganizadas, debido a la coyuntura nacional iniciada el 18 de octubre del 2019 denominada "estallido social", de la cual surgieron manifestaciones que alteraron el desarrollo normal de las actividades en distintos sectores de la sociedad. 
su historia familiar. Posteriormente, esa información se le entregó al equipo educativo.

- TAREA 7. Intercambio de tópicos que aluden a la diversidad cultural en el aula. De las entrevistas realizadas al equipo educativo derivaron los cuatro tópicos: ¿qué es diversidad cultural?, educación intercultural, implicaciones prácticas sobre cómo trabajar la educación intercultural y crianza.

- TAREA 8. Presentación de fondos de conocimiento. Debido al antecedente de que en el centro educativo se realizan visitas a los hogares, se decidió aprovechar esta instancia como una oportunidad de profundizar en el conocimiento de las culturas de las familias. Por esta razón se realizó una reunión con la educadora a cargo del nivel para presentarle la estrategia de los fondos de conocimiento, a fin de que ella, en una próxima visita, pudiera realizarlo. Se le otorgó una infografía para que pudiera acceder a la información y se discutió la temática; esta quedó de aplicarla en la siguiente visita y de compartirla con el equipo educativo para que todas sus integrantes pudieran acceder a este conocimiento.

Durante las fases y la realización de tareas se inició el proceso de evaluación de las acciones implementadas sobre la atención a la diversidad cultural, lo que se ve reflejado en los resultados que se presentarán a continuación. Estos resultados demostrarán las visiones del equipo educativo y las familias que participaron en la investigación.

Tal como se muestra en la figura 2, se utilizaron diferentes estrategias de recolección de información en las distintas fases de la investigación-acción. En primer lugar, se empleó la técnica de observación participante y, para registrar la información, se utilizaron registros abiertos, de tipo narrativo-descriptivo, registrada en vivo en las notas de campo (Latorre, 2005). Seguidamente, para conocer las perspectivas de los miembros de la comunidad educativa, se utilizó la técnica de entrevista semiestructurada con una guía de preguntas 
orientadoras (Hernández et al., 2014), la cual fue construida por medio de categorías apriorísticas con subcategorías, lo que constituye, orienta y direcciona el instrumento con el cual se recopila información (Cisterna, 2005).

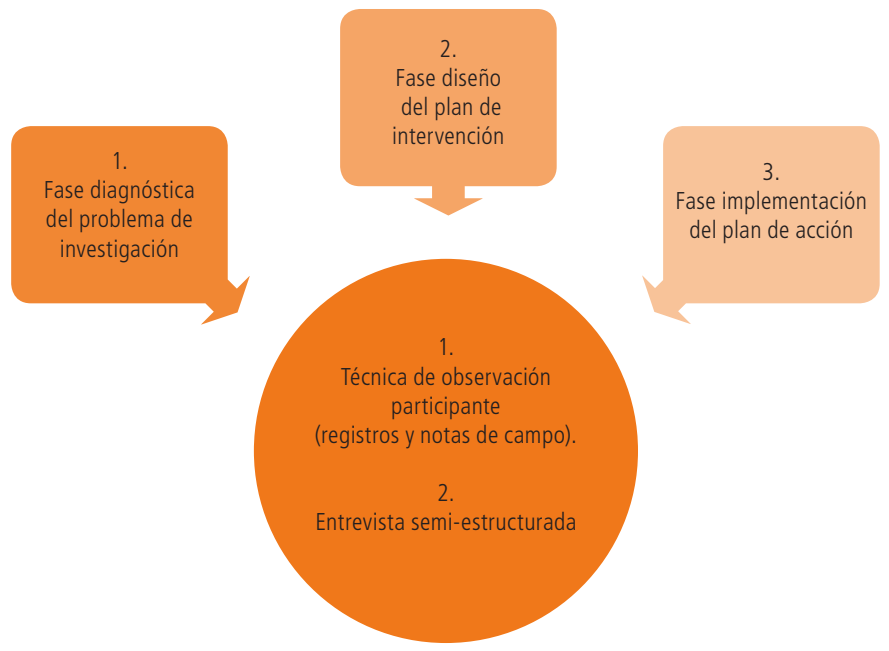

Fuente: Elaboración propia a partir de Sandin (2003)

En cuanto al rigor científico de la investigación cualitativa desarrollada, esta demostró su validez garantizando que la información que se obtuvo responde al fenómeno social estudiado. En la investigación se utilizaron diferentes fuentes y métodos de recolección de información, lo que se denomina triangulación de datos, para ver su grado de correspondencia (Hernández et al., 2014). Para ello se consultaron diversos informantes, los que cumplieron con diversos roles. Esto permitió mirar en toda su complejidad la problemática estudiada y generar datos provenientes de diversas fuentes. Adicionalmente, se hizo combinando diferentes instrumentos, como la observación 
participante y la entrevista semiestructurada, lo que ofreció la posibilidad de triangularlos.

Sobre las consideraciones éticas de la investigación, este estudio cualitativo ameritó que se estuviera por un tiempo prolongado en el espacio educativo, específicamente durante dos semestres académicos. De la misma forma, requirió interacción con los participantes del fenómeno de estudio, con cercanía a la realidad estudiada y, fundamentalmente, el trabajo directo con personas. Estas condiciones demandan una consideración de aspectos éticos por parte de los investigadores.

En relación con el consentimiento informado, se le reconoce como un código ético que inicialmente se implementó en el campo biomédico, por la información y autorizaciones que deben solicitárseles a los pacientes. Su adaptación a los estudios humanísticos y sociales es más reciente. Aguilera-Guzmán et al. (2008) lo definen como un proceso social que brinda información sobre la investigación de manera comprensible para el sujeto, por lo que le permitirá asegurarse de que se entienda la información, que pueda resolver dudas y que haya opción para que se niegue a participar o para manifestar su voluntad para colaborar sin que se le haya intimidado, influido o dado algún incentivo indebido.

Además, se entregó por escrito un consentimiento informado al equipo educativo y a las familias, que autorizaba la participación en esta investigación.

\section{RESULTADOS}

En esta sección se presentará una aproximación a los datos otorgados por los participantes en relación con la atención a la diversidad cultural, considerando las perspectivas del equipo educativo y de las familias que forman parte del aula, a partir de 5 temas de análisis.

\section{Prácticas de atención a la diversidad cultural}


Se refiere a si existen prácticas de parte del equipo educativo que atiendan a la diversidad cultural presente en el aula; por ello es que se les consultó a los participantes desde su concepción teórica y sobre las prácticas concretas.

Respecto de la concepción teórica sobre la diversidad cultural, declararon que esta diversidad solo se da en personas que tienen origen en otro país, lo cual niega el reconocimiento de la diversidad cultural propia de los chilenos provenientes de diversas regiones o sectores, cargados con historias de vida que también ameritan ser atendidas.

"Aceptar culturas de diferentes países" (Asistente técnico 2, Entrevista [E], fase 1).

"Aceptar el origen de cada país" (Asistente técnico 1, [E], fase 1).

Además, cuando se conceptualiza esta diversidad solo se refieren a "ellos" (ciudadanos haitianos) y no al resto de las familias presentes en el aula, lo cual demuestra la invisibilización de las diferencias culturales en sala. Estas respuestas se asocian a lo observado y registrado en las notas de campo, debido a que en ocasiones se presenciaron comentarios del equipo educativo que aludían a los ciudadanos haitianos como un problema y se les atribuían ciertas características como "brutos", "pesados" y "raros".

La educadora del nivel conceptualiza la diversidad cultural desde una mirada diferente, ya que verbaliza que la diversidad:

"Somos todos, no solo los países, porque vivimos cosas distintas" (Educadora, E, fase 1).

Sobre este particular, vale acotar que se observa inconsistencia, pues esta mirada que reconoce la complejidad del concepto no concuerda con las observaciones mencionadas, ya que esta también tomó parte en los comentarios mencionados. A su vez, las familias dieron opiniones divididas acerca del conocimiento del equipo educativo sobre las diferencias culturales. 
"No creo que las conozcan muy bien" (Madre 1, E, fase 1).

"Yo creo que sí las conocen bien" (Madre 2, E, fase 1).

Por otra parte, se le consultó al equipo educativo cómo se atendía la diversidad cultural en el nivel y qué prácticas identificaban como interculturales. Ante esto, todos los miembros del equipo coincidieron en que no creían que realizaran prácticas interculturales.

"Se han hecho pocas cosas para la atención a la diversidad; solo una con las comidas. Es que igual las familias extranjeras tienen poco tiempo para realizar actividades, priorizan su trabajo" (Asistente técnico 3, E, fase 1).

"No se trabaja, se hace lo mismo con todos, no se hacen diferencias" (Educadora, E, fase 1).

Si bien la educadora considera que no hacer diferencias es positivo, al no reconocer las diferencias culturales no se puede propiciar un espacio de tolerancia, valoración y respeto. Esto coincide con las observaciones realizadas, ya que el mayor problema evidenciado es que no se llevan a cabo prácticas de atención a la diversidad cultural en el aula. Las familias concuerdan en que no saben cómo se atiende las diferencias culturales presentes en el aula, lo que revela dificultades para hacer efectiva la participación de las familias en los centros educativos, y los canales de comunicación entre la familia y la escuela siguen reproduciendo esquemas tradicionales.

\section{Implicaciones prácticas de la atención a la diversidad cultural}

A partir de las entrevistas realizadas, se reveló que el equipo conoce las siguientes estrategias para la atención a la diversidad: "hacer música", "hacer degustaciones de la comida" y "fiestas". Sus dudas sobre la temática se asociaban a que no sabían cómo manejar acciones 
prácticas, que les complicaba el idioma y que no creían que se podría trabajar con los niños y niñas del nivel, por lo que desde ahí solicitaron que para el plan de intervención se integrara esa información hacia ellas como equipo.

"No sé, es que tampoco les entiendo mucho; entonces no sabría qué hacer con el idioma y eso. Pero podríamos hacer más fiestas y degustaciones de las comidas" (Asistente técnico 2, E, fase 2).

"Yo creo que se puede trabajar haciendo música, igual ellos son chiquititos. Me gustaría saber cómo se hace para poder ayudarlos" (Asistente técnico 1, E, fase 2).

"Ellos deberían tener un espacio donde tengan materiales; por ejemplo, instrumentos musicales de ellos hacen falta. Es que igual, si uno presenta un tambor, ellos no entenderán mucho, pero sí niños más grandes" (Asistente técnico 3, E, fase 2).

Estas débiles aproximaciones del equipo educativo a la atención de la diversidad se asocian a que la formación de los educadores en el ámbito de la educación intercultural ha sido escasa y no siempre adecuada (Verdeja, 2018). Lo señalado anteriormente corresponde directamente con lo observado en la práctica, ya que no se evidenciaron acciones significativas para la atención de la diversidad cultural del aula.

El equipo educativo coincide en que hace falta mayor información respecto de cómo responder a la diversidad cultural del aula a través de estrategias, por lo que solicitan que se les hagan llegar implicaciones prácticas para atender a la diversidad.

\section{Visibilidad y valoración de prácticas de crianza, tradiciones y valores de las familias}

La percepción de caso todo el equipo educativo demostró que creían que era importante darles visibilidad a las familias. 
"Porque son los primeros educadores" (Educadora, E, fase 2).

"Porque es importante que ambas partes nos conozcamos; ponle que igual en la adaptación nos dimos cuenta que son brutos" (Asistente técnico 1, E, fase 2).

"En el nivel, los padres siento que no han sido comprometidos en distintos aspectos, no demuestran su cultura, no tienen ganas por trabajo, o por tiempo, no se dan instancias para que puedan proponer, asisten poco.... Para empezar, ellos deberían comprometerse con sus hijos y hacer trabajos más profundos; si tiran al niño pa’ adentro todas" (Asistente técnico 3, E, fase 2).

Es relevante considerar que la crianza no solo se da en el hogar, sino que también en los centros educativos, sobre todo en este nivel; es por esto que la responsabilidad de la crianza es compartida y deberían estar articulados la escuela y la familia para favorecer el desarrollo de los niños y niñas. Es imprescindible que exista comprensión de los escenarios en que se generan las prácticas de crianza, tanto de las familias como de los profesionales en educación, para generar mejores contextos (Bravo, 2018). Por eso es importante que se asuma un rol por parte de ambos agentes y no se le traspase la responsabilidad total al otro; los docentes deberían tener la capacidad de generar estrategias para responder a este desafío. Según lo observado en el aula, en reiteradas ocasiones se evidenciaron comentarios que aludían a aspectos de la crianza que no entendían o que sentían que se deberían mejorar por parte de las familias, pero se enfocaban en la crítica y no en acciones para remediarlos y/o acompañarlos. Las familias, por su parte, mencionaron que era importante que se valoren sus prácticas de crianza, tradiciones y valores, para que haya una cohesión entre ambas partes ante el cuidado de sus hijos/as.

"Creo que es importante, porque así nos conocen más a nosotros, las cosas que hacemos y de dónde venimos” (Madre 3, E, fase 2). 
"Sería bueno que nos conocieran más a los papás, así nos unimos para hacer lo mismo que nosotros hacemos en la casa" (Madre 4, $\mathrm{E}$, fase 2).

Un ejemplo concreto, como el desconocimiento del lugar del que provienen las familias, supone no considerar su lengua y su cultura, lo cual podría generar una ruptura para los niños y niñas con las tradiciones de la comunidad, además de ocasionar el riesgo de que no terminen dominando su propia lengua, aspecto que debe evitarse a toda costa (Montón, 2003).

\section{Relación familia-centro educativo}

En cuanto al rol de las familias en el aula, una de las personas del equipo educativo, ante la pregunta de qué rol cumple la familia en el aula y cómo se podría incorporar para trabajar las diferencias culturales, mencionó que:

"La enseñanza viene de la casa, no es muy compartido el aprendizaje, lo que hacemos con los hijos... Me gustaría que un papá vea la rutina, cómo mudamos, y que también alguna vez ellos vinieran a contarnos cómo son los cuidados que ellos hacen" (Asistente técnico 2, E, fase 2).

"Hay un problema con el rol; es un tema de fondo porque aquí no explicamos la metodología y se debería empezar desde la matrícula, porque si estamos recibiendo a tantas personas hay que acogerlos po'” (Asistente técnico 3, E, fase 2).

Las familias, por su parte, coinciden en que no saben cómo podrían participar dentro del aula, situación que debería cambiar debido a la importancia de la relación entre la familia y la escuela, ya que durante los tres primeros años de vida se hace necesario gestionar estrategias 
en común en beneficio de la continuidad de las prácticas de crianza de las familias (Gabbins, 2016).

Con respecto al rol del equipo educativo ante la atención a la diversidad cultural, señalan que se relaciona con:

"Querer, incluir, amar e integrar a todos" (Asistente técnico 2, E, fase 2).

"Acoger a las familias en cuanto a su culturalidad" (Asistente técnico 3, E, fase 2).

"Tener conocimiento y estar informados de ellos y sus culturas" (Educadora, E, fase 2).

Su percepción se asocia a lo que señala la educación intercultural, basada en la diversidad, ya que nos permite comprender que no se puede definir a las personas en función de sus características estáticas, sino en función de las diferentes formas de ver, estar y construir el mundo, la realidad y las relaciones con los demás (Peñalva y Soriano, 2010). En sus intervenciones, parecieran recoger la combinación de aspectos actitudinales, conceptuales y procedimentales; sin embargo, llama la atención que esta realidad no se ha evidenciado en los registros realizados en las notas de campo, ya que, por ejemplo, se constató que "una mañana Martín" , hijo de padres de nacionalidad haitiana, llega al aula y su madre lo deja con el equipo educativo y se retira. Una de las asistentes técnicas le quita el gorro que traía y verbaliza: “Qué le hicieron en la cabeza? ¿Por qué le hacen estas "cosas"? (refiriéndose a las trenzas) En vez de preocuparse de otras cosas...”( $\left.\mathrm{NC} \mathrm{1}^{3}\right)$. Este comentario demuestra un prejuicio por parte de una de las personas del equipo educativo, ya que hace alusión a que la madre,

2 Nombre ficticio con el fin de resguardar la identidad de los participantes de la investigación.

3 Nota de campo. 
al hacerle trenzas, estaría dejando de lado otros quehaceres, que se considera más importantes, respecto de su hijo.

\section{Acciones implementadas de atención a la diversidad cultural}

En cuanto a la apreciación sobre las acciones desarrolladas para la atención a la diversidad, se consultó en las entrevistas sobre su calidad, pertinencia e innovación. Los participantes coincidieron en que se logró dar una mayor visibilidad a las culturas en el aula.

"Se incluyó información que nosotras como equipo no podríamos haber hecho; está todo a la vista” (Asistente técnico 2, E, fase 3).

"Cada cosa contribuye a mejorar, aunque siempre habrá más estrategias que irán surgiendo” (Asistente técnico 3, E, fase 3).

Esto permite recoger el reconocimiento de parte del equipo educativo de que las acciones buscaron contribuir a la realidad educativa del aula, haciendo aportes para atender a la necesidad presentada. En cuanto a lo que creyeron que faltó por implementar u otras acciones necesarias de introducir, señalaron:

"Ferias gastronómicas" (Madre 4, E, fase 3).

"Mediador cultural" (Educadora, E, fase 3).

"Una charla con las familias" (Madre 5, E, fase 3).

Sobre este punto se infiere que hay un interés por aplicar otros tipos de acciones, además de los presentados, proponiendo opciones que les gustaría haber evidenciado durante el proceso de investigación.

Finalmente, con respecto a la pertinencia, todos los participantes coincidieron en que sí lo fueron, y en particular destacaron como relevantes las acciones referidas al diccionario creol-español y al cuaderno viajero. 
"El diccionario creol-español, ya que nos permitió a nosotras poder comunicarnos mejor con las familias y conocer palabras de su idioma" (Asistente técnico 3, E, fase 3).

"Cuaderno viajero, porque es una iniciativa importante de considerar en la sala; pudimos escribir de nosotros como familias y de nuestros hijos y eso se valora" (Madre 6, E, fase 3).

A partir de los puntos analizados desde el proceso de valoración de los participantes, se puede reflexionar críticamente sobre el alcance de la investigación, ya que se partió visualizando las concepciones que tenía el equipo educativo sobre la diversidad cultural. Estas creencias solo contemplaban a las personas migrantes, sin considerar que las familias chilenas también poseen diversas características que deberían tomarse en cuenta a la hora de atender a los niños y nińas. Si bien se asumió desde un comienzo que el proceso de investigación-acción iba a ser acotado, porque solo se llevó a cabo un espiral de cambio, lo que no es suficiente para generar transformaciones profundas a nivel de percepción de las personas involucradas, sí se pudo evidenciar que el equipo educativo finalmente reconoció acciones y valoró los aportes entregados desde esta investigación.

Varias de las acciones sufrieron modificaciones y fueron acotadas, por lo que su integración se realizó en un periodo muy breve, lo que dificultó la visualización de cambios significativos, como se mencionó anteriormente. Es por esto que la valoración de los participantes se fundó en sus creencias personales de acuerdo a lo presentado y algunas de las intervenciones no fueron consideradas significativas, ya que, por ejemplo, se decidió hacer una intervención dentro del panel del aula y eso no muchos padres lo pudieron constatar.

\section{CONCLUSIONES}

El trabajo de investigación presentado tuvo como objetivo analizar el proceso de atención pedagógica ofrecida a la diversidad cultural 
del nivel sala cuna menor; en específico se buscó identificar prácticas de atención pedagógica a la diversidad cultural por parte del equipo educativo, diseñar acciones que favorecieran la integración de la diversidad cultural y evaluar dichas acciones. En este trabajo se considera la diversidad cultural como parte de un marco social democrático y de relaciones entre distintas culturas, y su reconocimiento se configura desde las construcciones de sentido que se generan en los procesos de socialización primaria (Ibáñez-Salgado et al., 2012).

En función de lo anterior, en un comienzo el estudio identificó que no se llevaban a cabo prácticas de atención pedagógica a la diversidad cultural del aula; asimismo, el equipo educativo reconoció esta carencia, además de que creían que no era posible intervenir con niños/as de esta edad. Respecto de esta última declaración, EscolanoPérez et al. (2014) revelan que las habilidades de los niños/as han sido subestimadas a lo largo de los años. Las familias también dieron cuenta de aquello, ya que no sentían que su cultura se viera reflejada dentro del espacio educativo.

La percepción inicial de los participantes se asoció a que veían la diversidad cultural como propia de las familias migrantes, sin reconocer que todas las personas poseen una cultura distinta, lo que refuerza la idea del estudio realizado por Barrero y Rosero (2018), el cual da cuenta de las diversas concepciones de los educadores en educación inicial; en esta investigación, la mayor parte de los participantes identificó la diversidad con la condición de extranjeros e inmigrantes de los estudiantes. Luego de realizar dicho diagnóstico, se decidió proponer acciones para favorecer el proceso de atención, por lo que se diseñaron e implementaron ocho tareas. A partir del proceso de su implementación, puede concluirse que las acciones fueron pertinentes para los participantes, como un primer espacio de acercamiento al proceso de atención a la diversidad cultural, ya que reconocieron que fueron importantes para poder conocer más sobre la temática y cómo abordarla, lo que permitió dar cumplimiento a los objetivos fijados en la investigación. 
Uno de los cambios principales que tuvieron los participantes respecto de las acciones implementadas fue la valoración de la atención de la diversidad cultural, ya que pudieron reconocer que va más allá de las personas migrantes; y también su repercusión, al trabajarla con los niños/as y familias. También se logró que las familias hicieran un reconocimiento positivo de las acciones como instancias importantes para darles visibilidad a ellos dentro del aula. Las acciones implementadas parten desde la base de que la diversidad cultural está presente en todas las aulas, por lo que se hace necesario fortalecer los procesos de profesionalidad docente en un desafío político educativo (Mora, 2018).

Se destaca que el desafío docente actual es conocer el camino de la educación intercultural, ya que esta busca el enriquecimiento mutuo entre grupos humanos diversos y el reconocimiento y valoración de la diversidad cultural dentro de la sociedad y en el sistema educativo (Aranda, 2011). Esto aporta una nueva visión de la educación, que visibiliza, valora y respeta la diversidad, considerándola como una riqueza.

Si bien se reconoce que se pudieron cumplir los objetivos planteados, se sugiere dar continuidad a las acciones para poder realizarlas con una incidencia más profunda. Se considera el tiempo como un factor limitante dentro de la investigación, ya que el lapso de trabajo impidió profundizar dichas acciones, lo que habría enriquecido el espacio de intercambio y de evaluación sistemática de cómo repercutieron en los participantes.

El desafío principal que se encontró en la investigación fue el aporte de las tareas realizadas; sin embargo, estas no fueron totalmente suficientes y sería necesario realizar un nuevo diagnóstico (en el espiral de cambio) para llevar a cabo un nuevo proceso de investigaciónacción que pueda mejorar los aspectos limitantes, como en este caso lo fueron el tiempo y la profundidad de las acciones realizadas. De ahí que se hizo una retroalimentación y propuesta para las acciones realizadas con el equipo educativo y las familias. 
Finalmente, se requiere poder llevar a cabo las acciones que quedaron inconclusas, por lo que se propone concretar la búsqueda de la figura de un mediador cultural, aplicar técnicas de fondos de conocimiento (aparte de la visita a los hogares) propuesta por Esteban-Guitart et al. (2012), profundizar la temática de trabajo directo con los niños y niñas del aula y estructurar acciones en un periodo de tiempo prolongado, que permitan realizar los espirales de investigación-acción completos.

\section{REFERENCIAS}

Aguilera-Guzmán, R., Mondragón, L. y Medina-Mora, E. (2008). Consideraciones éticas en intervenciones comunitarias: la pertinencia del consentimiento informado. Salud Mental, 31(2), 129-138.

Aranda, V. (2011). Reflexión y análisis de políticas y prácticas innovadoras a la luz de las representaciones sociales y de la necesidad de una educación intercultural en la formación inicial docente. Estudios Pedagógicos, 37(2), 301-314. http://dx.doi.org/10.4067/ S0718-07052011000200018

Arroyo, M. (2013). La educación intercultural: un camino hacia la inclusión educativa. Revista de Educación Inclusiva, 6(2), 144-159. https://revistaeducacioninclusiva.es/index.php/REI/article/view/186

Barrero, A. y Rosero, A. (2018). Estado del arte sobre concepciones de la diversidad en el contexto escolar infantil. Revista Latinoamericana de Educación Inclusiva, 12(1), 39-55. http://dx.doi.org/10.4067/ S0718-73782018000100039

Barrios, L. y Palou, B. (2014). Educación intercultural en Chile: la integración del alumnado extranjero en el sistema escolar. Revista Educación y Educadores, 17(3), 405-426. http://dx.doi. org/10.5294/edu.2014.17.3.1 
Bernabé, M. (2012). Pluriculturalidad, multiculturalidad e interculturalidad, conocimientos necesarios para la labor docente. Revista Educativa Hekademos, 11(5), 68-76. https://roderic.uv.es/ handle/10550/47898

Bravo, A. (2018). Prácticas de crianza en la sala cuna y en el hogar: un estudio de caso. Educación, 27(53), 7-23. http://dx.doi. org/10.18800/educacion.201802.001

Cano, V. y Soffia, M. (2009). Los estudios sobre migración internacional en Chile: apuntes y comentarios para una agenda de investigación actualizada. Papeles de Población, 15(61), 129-167. http://www.scielo.org.mx/scielo.php?script=sci_arttext\&pid=S140574252009000300007\&lng=es\&nrm=iso

Chávez, A., Rodríguez, J., Acuña, M., Barquero, J., Macadar, D., Pinto, J. y Sobrino, J. (2016). Migración interna y cambios metropolitanos: ¿Qué está pasando en las grandes ciudades de América Latina? Revista Latinoamericana de Población, 10(18), 7-41. https://doi.org/10.31406/relap2016.v10.i1.n18.1

Cisterna, F. (2005). Categorización y triangulación como procesos de validación del conocimiento en investigación cualitativa. Theoria, 14(1), 61-71. https://www.redalyc.org/ pdf/299/29900107.pdf

Consejo Nacional de la Cultura y las Artes (2014). Legislación cultural chilena. Publicaciones Cultura. https://www.cultura.gob.cl/wpcontent/uploads/2014/03/libro-legislacion-cultural.pdf

Consejo Nacional de la Cultura y las Artes (2016). Interculturalidad $y$ migración. Gobierno de Chile. https://www.cultura.gob.cl/ wp-content/uploads/2017/07/libro_seminario_interculturalidad_ migracion.pdf 
Cuello, C. (2016). El profesor como mediador intercultural en la sala de clases: diseño de un programa de desarrollo de competencias interculturales orientado a profesores de colegios anfitriones participantes de programas de AFS Chile [tesis de maestría]. Pontificia Universidad Católica de Chile. https://repositorio.uc.cl/handle/11534/21261

Echeverría, G. (2005). Análisis cualitativo por categorías. Universidad Academia de Humanismo Cristiano. Escuela de Psicología.

Escolano Utrilla, S., Ortiz Véliz, J., y Moreno Mora, R. (2018). Dinámica y estructura de las migraciones residenciales interurbanas en Chile, 1987-2002. Revista INVI, 33(94), 105-133. http://dx.doi. org/10.4067/S0718-83582018000300105.

Escolano-Pérez, E., Gaeta-González, M. y Herrero-Nivela, M. (2014). Desarrollo y uso de habilidades metacognitivas infantiles: secuencias observacionales. Revista INFAD de Psicología. International Journal of Developmental and Educational Psychology, 5(1), 453-462. https:// doi.org/10.17060/ijodaep.2014.n1.v5.705

Esteban-Guitart, M., Oller, J., \& Vila, I. (2012). Vinculando escuela, familia y comunidad a través de los fondos de conocimiento e identidad. Un estudio de caso con una familia de origen marroquí. Revista de Investigación en Educación, 10(2), 21-34. https://dialnet. unirioja.es/servlet/articulo?codigo $=4732917$

Fermín, M. (2007). Retos en la formación del docente de Educación Inicial: La atención a la diversidad. Revista de Investigación, 31(62), 071-092. https://www.redalyc.org/comocitar.oa?id=376140377004

Fermín, M. (2010). Atención a la diversidad en la infancia: una mirada desde la educación intercultural. En Retos Educativos en la Sociedad del Conocimiento (pp. 153-168). Davinci Continental.

Fermín, M. (2018). La diversidad cultural de los nińos del Amazonas venezolano. Alternativas de atención pedagógica. Revista Educación Superior y Sociedad (ESS), 18(18), 121-151. https://www.iesalc. unesco.org/ess/index.php/ess3/article/view/9 
Fermín-González, M. (2021). Formación de educadores de la primera infancia en competencias interculturales. En Caheiro-González, M., López-Gómez. E. y Domínguez-Garrido, M. (Ed.). Investigación e internacionalización en la formación basada en competencias (pp. 181-198). Editorial Dykinson.

Fernández, M. P. (2018). Mapa del estudiantado extranjero en el sistema escolar chileno (2015-2017). MINEDUC. https://www. mineduc.cl/wp-content/uploads/sites/19/2018/05/MAPA_ ESTUDIANTES_EXTRANJEROS_SISTEMA_ESCOLAR_ CHILENO_2015_2017.pdf

Fuenzalida, D., Coimin, B., Lundy, E. y Galaz, D. (2017). Hablemos español, hablemos creol: Cuadernillo creol haitiano-español. UAH. https://www.uahurtado.cl/wp-images/uploads/2017/01/ annpalepanyol-1.pdf

Fundación Integra (2018). Referente Curricular de Fundación Integra. INTEGRA. http://web.integra.cl/web_integra//uploads/202\%20 -\%20ANEXO\%206\%20Referente\%20Curricular\%202019\%20 Borrador.pdf

Gomila, M. y Pascual, B. (2015). La participación de las familias en el sistema educativo: la percepción del profesorado en formación. Revista Electrónica Interuniversitaria de Formación del Profesorado, 18(3), 99-112. https://doi.org/10.6018/reifop.18.3.199321

Grupo Consultivo de Atención y Desarrollo de la Primera Infancia. (2016). Informe mundial sobre equidad y la primera infancia. Consultative Group on Early Childhood Care and Development (CGECCD). http://equidadparalainfancia.org/2016/08/informemundial-sobre-equidad-y-la-primera-infancia/

Gubbins, V. y Ibarra, S. (2016). Estrategias educativas familiares en enseñanza básica: análisis psicométrico de una escala de prácticas parentales. Psykhe, 25(1), 1-17. https://doi.org/10.7764/ psykhe.25.1.773 
Hernández, R., Fernández, C. y Baptista, M. (2014). Metodología de la investigación. McGraw-Hill.

Ibáńez-Salgado, N. (2015). La diversidad en la construcción de mundo de niños y niñas de dos culturas. Revista Latinoamericana de Ciencias Sociales, Niñez y Juventud, 13(1), 357-368. http:// dx.doi.org/10.11600/1692715x.13121260914

Ibáñez-Salgado, N., Díaz-Arce, T., Druker-Ibáñez, S. y RodríguezOlea, M. (2012). La comprensión de la diversidad en interculturalidad y educación. Revista de Ciencias Sociales, 19(9), 215-240. http:// www.scielo.org.mx/scielo.php?script=sci_arttext\&pid=S1405$14352012000200009 \& \operatorname{lng}=$ es\&nrm=iso

Instituto Nacional de Estadística (2017). Sintesis resultados Censo 2017. Chile. https://www.censo2017.cl/descargas/home/sintesisde-resultados-censo2017.pdf

Instituto Nacional de Estadística (2018). Radiografía de género: pueblos originarios en Chile. Unidad de estudios y estadísticas de género. https://historico-amu.ine.cl/genero/files/estadisticas/pdf/ documentos/radiografia-de-genero-pueblos-originarios-chile2017.pdf

Junta Nacional de Jardines Infantiles. (2009). La perspectiva intercultural en la práctica educativa. JUNJI.

Latorre, A. (2005). La investigación-acción: conocer y cambiar la práctica educativa. Graó.

Llugain, C. (2013). La construcción del vínculo familia-escuela en el ingreso a educación inicial: estudio de caso en un jardín de infantes. En V Congreso Internacional de Investigación y Práctica Profesional en Psicología, XX Jornadas de Investigación Noveno Encuentro de Investigadores en Psicología del MERCOSUR. Buenos Aires, Argentina. https://www.aacademica.org/000-054/440 
Márquez, Y., Gutiérrez-Barroso, J. y Gómez-Galdona, N. (2017). Equidad de género y diversidad en la educación. European Scientific Journal, 13(7), 300-319. http://dx.doi.org/10.19044/ esj.2017.v13n7p300

Martínez, J., Cano, V. y Soffia, M. (2014). Tendencias y patrones de la migración latinoamericana y caribeña hacia 2010 y desafios para una agenda regional. CEPAL. https://repositorio.cepal.org/ handle/11362/37218

Ministerio de Educación (2017). El primer gran debate de la reforma educacional: La ley de inclusión escolar. Santiago, Chile: MINEDUC. https://bibliotecadigital.mineduc.cl/handle/20.500.12365/441

Ministerio de Educación (2018). Bases Curriculares Educación Parvularia. Santiago, Chile: MINEDUC. https://parvularia.mineduc.cl/ wp-content/uploads/sites/34/2018/03/Bases_Curriculares_Ed_ Parvularia_2018.pdf

Ministerio de Educación (2019). Marco para la buena enseñanza de Educación Parvularia. Santiago, Chile: MINEDUC. https:// parvularia.mineduc.cl/wp-content/uploads/sites/34/2019/08/ MBE_EP-Final.pdf

Mora, M. (2018). Política educativa para migrantes en Chile: un silencio elocuente. Revista Latinoamericana, 49, 31-257. http:// dx.doi.org/10.4067/S0718-65682018000100231

Moreno, I., Bermúdez, A., Ramos, J., Mora, C. y Torres D. (2016). Representaciones sociales de los maestros sobre la familia y su rol en la escuela. Encuentros, 14(1), 119-138. http://dx.doi. org/10.15665/re.v14i1.673

Naudon, P. (2016). Mujeres migrantes en Chile: significaciones sobre su rol de madre y la crianza de hijos. Revista Rumbos TS, (14), 99-112. http://revistafacso.ucentral.cl/index.php/rumbos/ article/view/37 
Peñalva, A. y Soriano, A. (2010). Objetivos y contenidos sobre interculturalidad en la formación inicial de educadores y educadoras. Estudios sobre Educación, 18, 37-57. https://revistas.unav.edu/index. php/estudios-sobre-educacion/article/view/4651

Ramos, C. (2015). Los paradigmas de la investigación científica. Avances en Psicología, 23(1), 9-17. https://doi.org/10.33539/avpsicol.2015. v23n1.167

Sandin, M. (2003). Investigación cualitativa en educación. Fundamentos $y$ tradiciones. McGraw-Hill.

UNESCO (2001). Declaración Universal de la UNESCO sobre la diversidad cultural. PRAXIS, (65-64), 279-284. https://www. revistas.una.ac.cr/index.php/praxis/article/view/4080

UNICEF (2020). Niños, niñas y adolescentes en Chile 2020. https:// www.unicef.org/chile/informes/ninos-ninas-y-adolescentes-enchile-2020

Verdeja, M. (2018). Percepciones del profesorado, alumnado y familias ante la diversidad cultural: el caso de un IES asturiano. Tendencias Pedagógicas, (31), 227-252. http://hdl.handle.net/10486/680844 